\title{
ARTHRODESIS OF THE CARPO-METACARPAL JOINT OF THE THUMB
}

\author{
Robert E. Carroll and Norman A. Hill, New York, United States of America \\ From Columbia Presbyterian Medical Centre and St Luke's Hospital Centre, New York
}

Sound arthrodesis of the carpo-metacarpal joint of the thumb can be difficult to achieve by the conventional technique of squaring off opposing joint surfaces at the proper angle and pinning them, even when bone grafts are added (Gervis 1949, Müller 1949, Leach and Bolton 1968, Eaton and Littler 1969). One reason for the difficulty in obtaining fusion is that when the saddle-shaped trapezium is simply planed off flat, there is only a thin disc of bone left for contact with the metacarpal diaphysis. The last series of carpo-metacarpal fusions published (Eaton and Littler 1969) had a 10 per cent incidence of pseudarthrosis, though not all the pseudarthroses were painful. They required three to four months in plaster after operation.

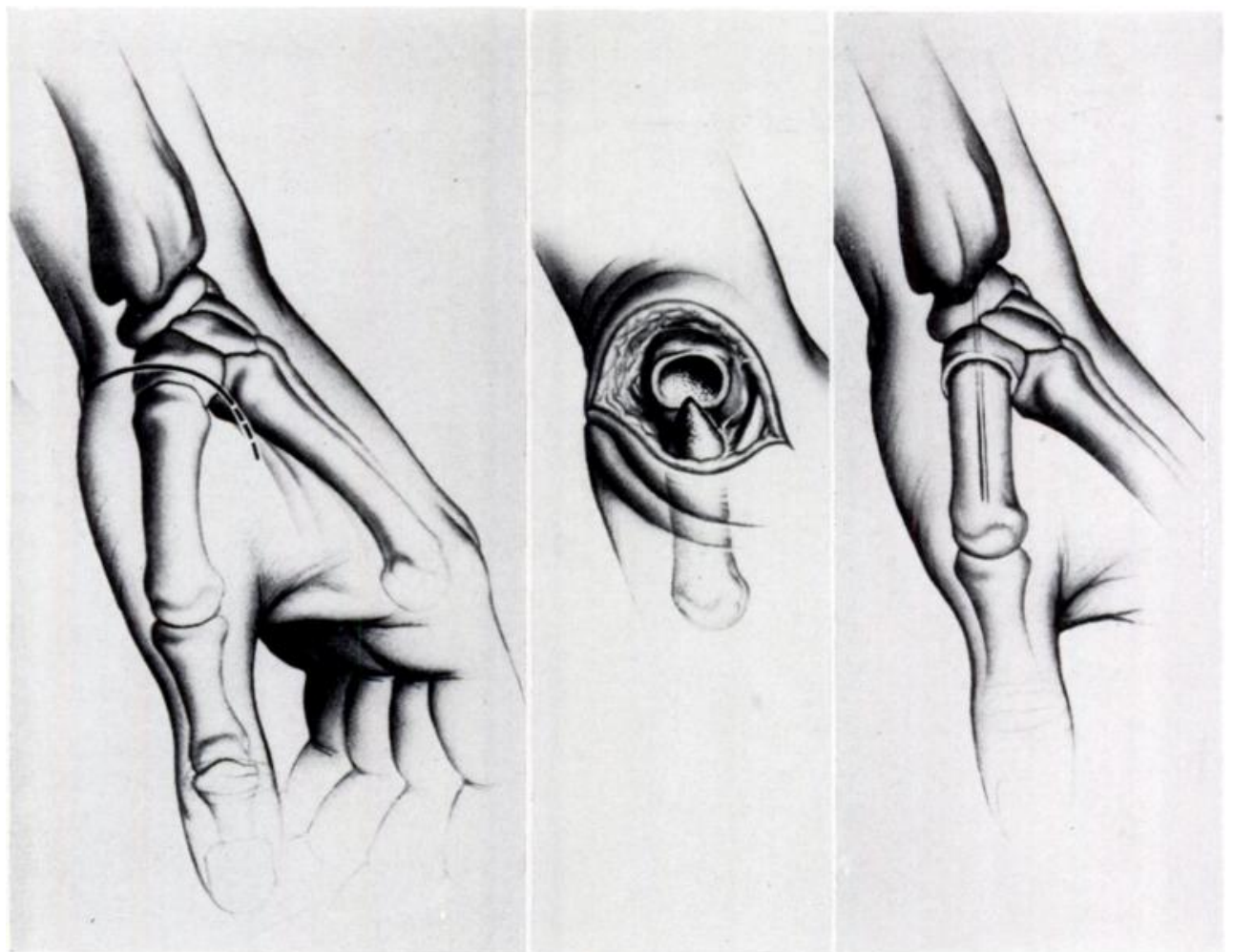

FIG. 1

Technique of trapezio-metacarpal arthrodesis.

\section{INDICATIONS}

Painful arthritis of the carpo-metacarpal joint can be treated conservatively. When this fails, surgical correction can be either by arthroplasty of the carpo-metacarpal joint or by arthrodesis. Arthroplasty gives an excellent range of painless motion, but the power of pinch is reduced. Arthrodesis permits a good range of thumb movement and excellent power of pinch. We therefore prefer arthrodesis provided the patient is less than fifty years old and shows no 
evidence of arthritis of the trapezio-scaphoid joint (Badger 1964). We also undertake arthrodesis to improve the position of the thumb in cases of paralysis with no tendons available for transfer, and in selected cases of cerebral palsy and arthrogryposis.

\section{TECHNIQUE OF OPERATION}

Our operative approach has evolved from a similar technique applied to other small joints in the hand (Carroll and Hill 1969) (Fig. 1). The trapezio-metacarpal joint is exposed by an incision over the dorsal aspect of the joint. The sensory branch of the radial nerve and the radial artery are preserved. After division of the capsular ligaments, the metacarpal base is dislocated into the wound. The articular cartilage and some cancellous bone are removed

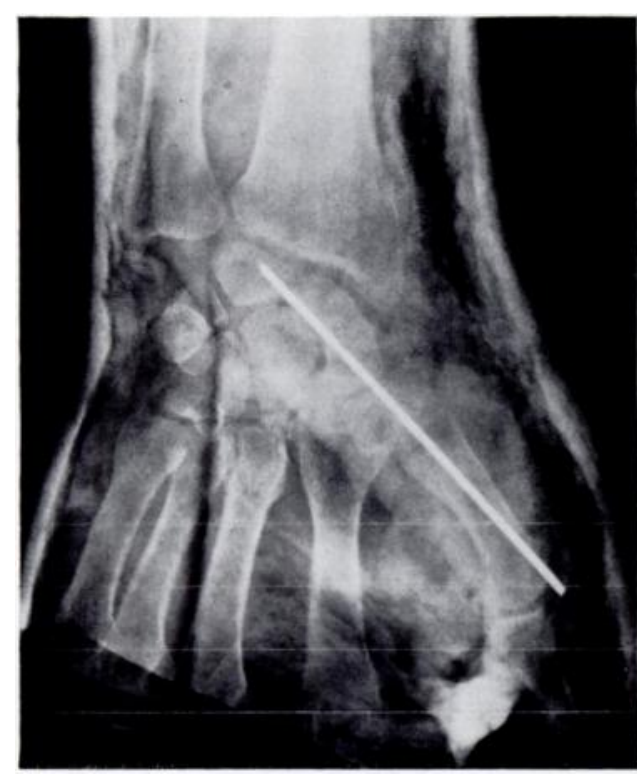

FIG. 2

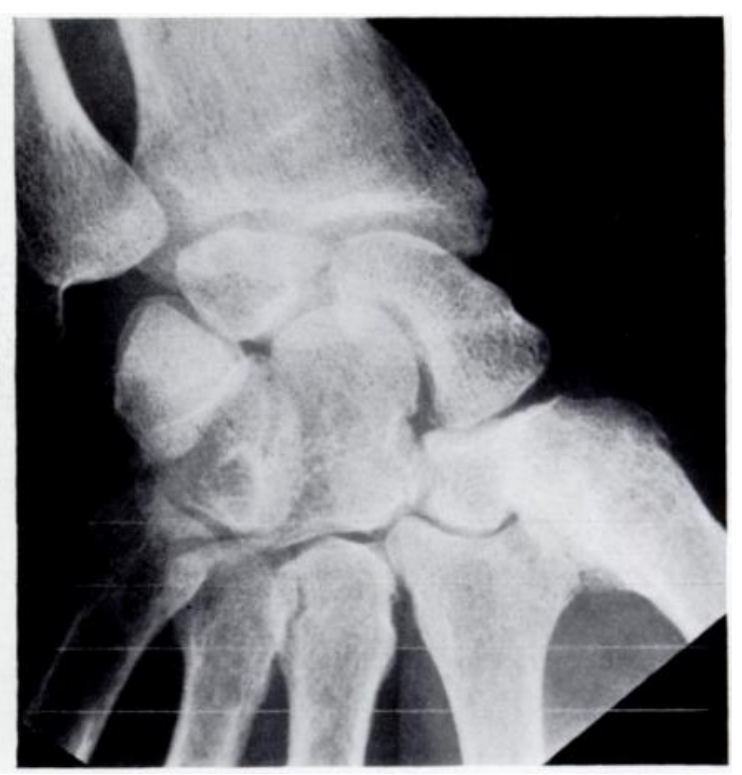

Fig. 3

Figure 2-Radiograph after operation, showing the arthrodesis site transfixed with a single wire. Figure 3Solid arthrodesis of trapezio-metacarpal joint.

with a rongeur and the proximal end of the metacarpal is fashioned into a smoothly rounded blunt point. It is important to complete this step first, because this allows wide exposure of the entire articular surface of the trapezium. A small round awl is used to make multiple holes around the rim of the trapezium at the osteo-cartilaginous junction. These awl holes are made one centimetre deep and at a 45-degree angle towards the centre of the articular cartilage, and are then connected with a small straight osteotome directed similarly towards the centre of the articular cartilage. Some surgeons prefer to do this with a power drill. This allows removal of the entire articular cartilage in one piece, leaving a cup-shaped receptacle for the metacarpal base. The walls of the cavity are cancellous, and afford excellent contact with the metacarpal base on all sides even when adjustments are made for proper metacarpal position. A retrograde Kirschner wire is then drilled out of the thumb metacarpal, the components are fitted together, and the wire is drilled on to transfix the joint. Bone grafts are not necessary. The usual position for the thumb metacarpal is $\mathbf{4 5}$ degrees from the plane of the index metacarpal in palmar abduction. Rotation is then corrected so that the pulp of the thumb opposes the fingers at an angle of 45 degrees of rotation. A forearm plaster is applied, including the thumb to its tip (Fig. 2). It takes an average of six weeks for these fusions to become sound, but eight to twelve weeks in patients with cerebral palsy (Fig. 3).

VOL. 55 B, No. 2, MAY 1973 


\section{RESULTS}

Sound fusion was obtained in thirty-six of thirty-nine patients. Of the three patients who developed pseudarthroses, two had severe cerebral palsy and one had arthritis. All three gained solid fusion after a second operation. There was one case of wound infection in a spastic patient who developed a pseudarthrosis.

Clinically, it is difficult to tell the difference between the thumb operated upon and the normal thumb in patients operated on for arthritis. All patients were able to adduct the thumb to touch the proximal phalanx of the index finger, and to oppose the thumb to the tips of all the fingers. Abduction from the plane of the palm lacked an average of 20 degrees when compared with the opposite thumb, but was quite adequate for grasp around relatively large objects such as jar lids. Pinch was equal to or better than that on the other side.

Cineradiographic studies in non-paralytic patients show that movement of the thumb metacarpal is quite restricted, averaging an arc of about 20 degrees in palmar abduction and adduction. The excellent range of movement that these patients showed clinically is due to a marked increase in mobility at the metacarpo-phalangeal joint and the trapezio-navicular joint when compared with the normal hand. Approximately 75 per cent of this increased range is at the metacarpo-phalangeal joint.

\section{SUMMARY}

1. A technique of arthrodesis of the trapezio-metacarpal joint of the thumb is described. Primary fusion was achieved in thirty-six of thirty-nine cases.

2. Compensatory movement at the adjacent joints permits a good range of thumb movement.

3. Trapezio-metacarpal arthrodesis is the operation of choice for patients under fifty with isolated osteoarthritis of this joint. It is also useful for stabilising the thumb in patients with paralysis of the thumb when adequate muscles for transfer are not available.

\section{REFERENCES}

BAdger, F. G. (1964): Arthrodesis of the Carpo-metacarpal Joint of the Thumb. Journal of Bone and Joint Surgery, 46-B, 162.

Carroll, R. E., and Hill, N. A. (1969): Small Joint Arthrodesis in Hand Reconstruction. Journal of Bone and Joint Surgery, 51-A, 1219.

EAtON, R. G., and Littler, J. W. (1969): A Study of the Basal Joint of the Thumb. Journal of Bone and Joint Surgery, 51-A, 661.

Gervis, H. (1949): Excision of the Trapezium for Osteoarthritis of the Trapezio-metacarpal Joint. Journal of Bone and Joint Surgery, 31-B, 537.

LEACH, R. E., and Bolton, P. E. (1968): Arthritis of the Carpo-metacarpal Joint of the Thumb. Journal of Bone and Joint Surgery, 50-A, 1171.

MüLlER, G. M. (1949): Arthrodesis of the Trapezio-metacarpal Joint for Osteoarthritis. Journal of Bone and Joint Surgery, 31-B, 540. 